\title{
Lactobacillus paracasei CNCM I-4034 and its culture supernatant modulate Salmonella-induced inflammation in a novel transwell co-culture of human intestinal-like dendritic and Caco-2 cells
}

Miriam Bermudez-Brito', Sergio Muñoz-Quezada', Carolina Gómez-Llorente', Esther Matencio², Fernando Romero ${ }^{2}$ and Angel Gil ${ }^{*}$

\begin{abstract}
Background: The action of probiotics has been studied in vitro in cells isolated from both mice and humans, particularly enterocytes (IECs), dendritic cells (DCs) and co-cultures of peripheral DCs and IECs. Peripheral DCs and murine DCs differ from human gut DCs, and to date there are no data on the action of any probiotic on co-cultured human IECs and human intestinal DCs. To address this issue, a novel transwell model was used. Human IECs (Caco-2 cells) grown in the upper chamber of transwell filters were co-cultured with intestinal-like human DCs grown in the basolateral compartment of the transwells. The system was apically exposed for $4 \mathrm{~h}$ to live probiotic L. paracasei CNCM I-4034 obtained from the faeces of breastfed infants or to its cell-free culture supernatant (CFS) and challenged with Salmonella typhi. The secretion of pro- and anti-inflammatory cytokines in the basolateral compartment was determined by immunoassay, and the DC expression pattern of 20 TLR signaling pathway genes was analysed by PCR array.
\end{abstract}

Results: The presence of the live probiotic alone significantly increased IL-1 $\beta$, IL-6, IL-8, TGF- $\beta 2$, RANTES and IP-10 levels and decreased IL-12p40, IL-10, TGF- $\beta 1$ and MIP-1a levels. This release was correlated with a significant increase in the expression of almost all TLR signaling genes. By contrast, incubation of the co-culture with CFS increased IL-1 $\beta$, IL-6, TGF- $\beta 2$ and IP-10 production only when Salmonella was present. This induction was correlated with an overall decrease in the expression of all TLR genes except TLR9, which was strongly up-regulated.

Conclusions: The data presented here clearly indicate that L. paracasei CNCM I-4034 significantly increases the release of pro-inflammatory cytokines, enhances TLR signaling pathway activation and stimulates rather than suppresses the innate immune system. Furthermore, our findings provide evidence that the effects of probiotics in the presence of IECS and DCs differ from the effects of probiotics on cultures of each cell type alone, as reported by us earlier. Thus, co-culture systems such as the one described here are needed to characterise the effects of probiotics in vitro, highlighting the potential utility of such co-cultures as a model system.

Keywords: Caco-2, Cytokines, Dendritic cells, Lactobacillus, Gene regulation, Pathogens, Probiotics

\footnotetext{
* Correspondence: agil@ugr.es

'Institute of Nutrition and Food Technology "José Mataix", Department of

Biochemistry and Molecular Biology II, University of Granada, Biomedical Research Center, Avenida del Conocimiento s/n, 18100 Armilla, Granada,

Spain

Full list of author information is available at the end of the article
} 


\section{Background}

Intestinal homeostasis is regulated by tight crosstalk between intestinal epithelial cells (IECs) and immune cells, especially dendritic cells (DCs) [1]. Signaling via innate pattern-recognition receptors (PRRs) such as Toll-like receptors (TLRs), NOD-like receptors (NLRs) and Ctype lectin receptors (CLRs) [2] directly influences the chemokine and cytokine response of DCs, as well as the crosstalk between the epithelium and immune cells in the lamina propria [3]. This signaling occurs largely through activation of the transcription nuclear factor kappa B (NF-kB) or mitogen-activated protein kinase (MAPK) pathways [4]. Furthermore, several studies have demonstrated that the typical phenotype of mucosal DCs can be obtained after co-culture with polarised epithelial cells, creating a "tolerogenic" environment $[5,6]$.

Probiotics are live microorganisms that have beneficial effects on the host. Although their mechanism of action is poorly understood [2], they are considered to be promising alternatives to antibiotics for the control and prevention of intestinal infections [7,8]. Lactobacillus paracasei CNCM I-4034 is a novel probiotic strain that was obtained from the faeces of breastfed infants and selected based on its probiotic properties, including adhesion to intestinal mucus, sensitivity to antibiotics and resistance to gastrointestinal juices, biliary salts, $\mathrm{NaCl}$ and low $\mathrm{pH}$ [9]. Previously, we reported that $L$. paracasei culture supernatant inhibits the growth of enterotoxigenic and enteropathogenic bacteria such as E. coli, Salmonella and Shigella [10].

In vitro studies of host immune responses have focused on probiotics and enterocytes or DCs. However, single cultures do not reflect the interactions occurring in the intestinal mucosa [11]. Thus, it is important to develop in vitro systems that include both DCs and IECs, both of which are crucial for intestinal homeostasis. Using a co-culture model including Caco-2 cells (IECs) and PBMCs, Haller et al. reported differential IEC activation by Escherichia coli and Lactobacillus strains [12]. However, PBMCs and murine DCs are quite different from human DCs [13]. Recently, using human intestinal-like DCs as a model, we reported the ability of L. paracasei CNCM I-4034 and its cell-free culture supernatant (CFS) to activate these immune cells [14]. Interestingly, these human DCs are Langerhans-like cells that extend dendritic processes and sample antigens similarly to the lamina propria DCs in the gut that sample luminal antigens [15]. We demonstrated previously that exposure to Salmonella enterica serovar typhi (S. typhi) led to reduced production of pro-inflammatory cytokines and chemokines in the presence of the probiotic and its supernatant. Both enhanced innate immune responses through the activation of TLR signaling pathway [14]. Our results coincide with those of another study indicating that live probiotic bacteria affect the intestinal immune response, whereas secreted components exert anti-inflammatory effects in the gastrointestinal tract [16]. Continue with this line, in the present work we investigated whether L. paracasei CNCM I-4034 or its CFS modulates the release of cytokines by intestinal-like DCs through a physical barrier of Caco2 cells and measured the effects that occur when the system is challenged with $S$. typhi. In addition, the expression pattern of selected TLR cascade signaling genes under these conditions was studied. The novel transwell model described here may better mimic the in vivo situation in which beneficial commensal probiotics interact with host immune cells via enterocytes.

\section{Methods}

\section{Ethic statement}

The ethical Committee of Granada's University approved this study. This study was conducted according to the guidelines laid down in the Declaration of Helsinki and the protocol using human DCs generated from umbilical cord blood $\mathrm{CD} 34^{+}$progenitor cells was approved by the Ethical Committee of the University of Granada.

\section{Design of the study}

A model was developed based on an in vitro transwell co-culture of IECs and human intestinal-like DCs. Caco-2 cells were cultured in the upper part of the transwell inserts, with human intestinal-like DCs in the lower chamber. L. paracasei CNCM I-4034 and S. typhi CECT 725, as well as CFS from $L$. paracasei, were added apically either alone or in combination (Figure 1). Negative control co-cultures were not exposed to any bacteria or CFS. As a positive control of TLR signaling pathway activation E. coli lipopolysaccharide (LPS) stimulation was used.

\section{Bacterial strains and culture conditions}

L. paracasei CNCM I-4034, which was selected for its immunomodulatory capacity, was isolated from the faeces of breast-fed newborns [9]. The probiotic strain was grown in De Man-Rogosa-Sharpe (MRS) broth medium (Oxoid, Basingstoke, United Kingdom) under anaerobic conditions at $37^{\circ} \mathrm{C}$. The bacterial cultures were grown overnight until they reached the stationary phase.

Salmonella typhi CECT 725 was provided by the Spanish Type Culture Collection (CECT; Burjassot, Spain). Salmonella was cultured for $8 \mathrm{~h}$ at $37^{\circ} \mathrm{C}$ in tryptone soy broth (Panreac Química, Barcelona, Spain) and then subcultured 1:500 in RPMI 1640 medium (Sigma-Aldrich) containing $10 \%$ foetal bovine serum (FBS; Gibco Invitrogen, Paisley, United Kingdom) at $37^{\circ} \mathrm{C}$ overnight. 


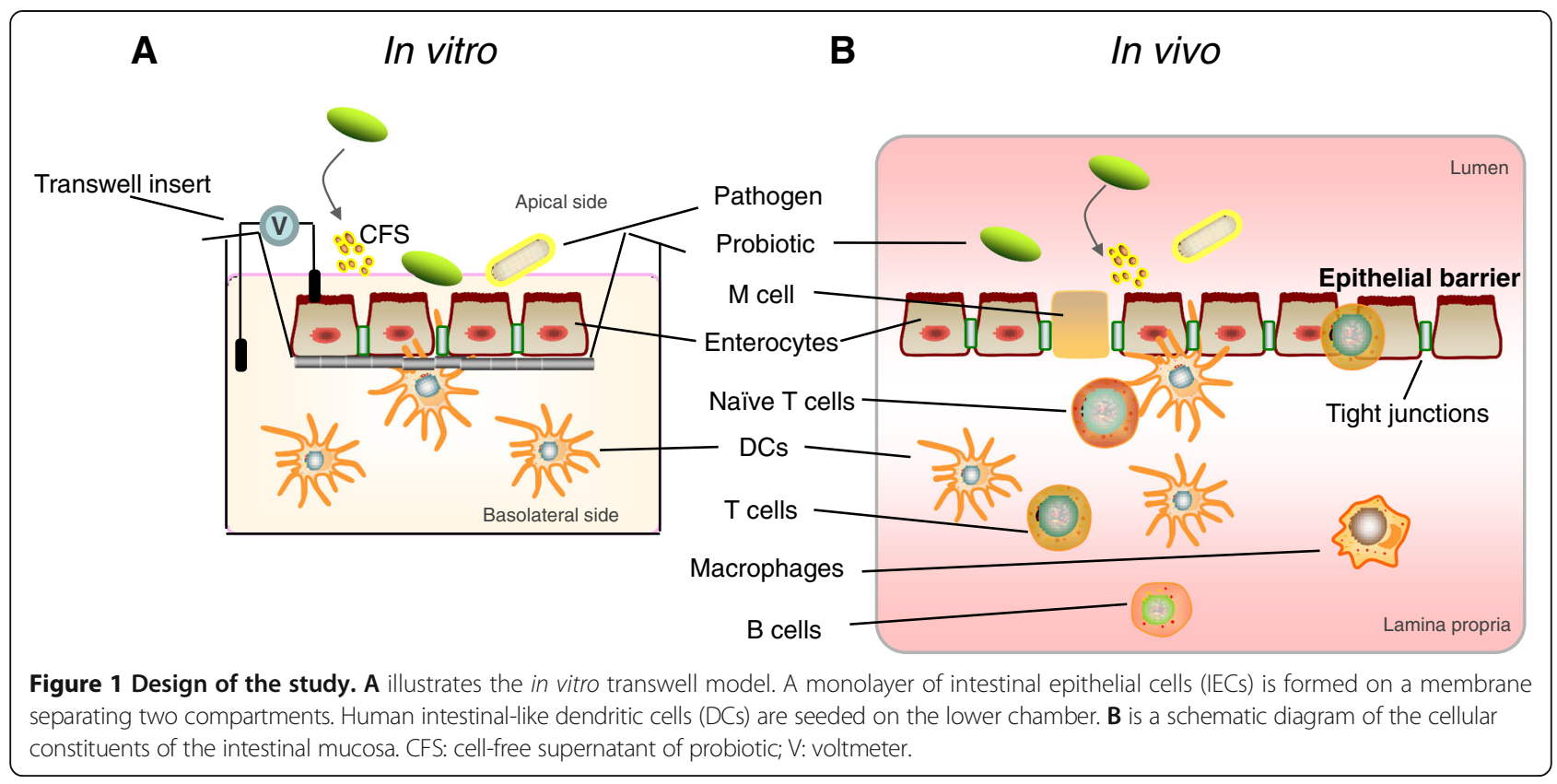

\section{Preparation of cell-free culture supernatant of $L$. paracasei CNCM I-4034}

The CFS was prepared as described by Bermudez-Brito et al. [14]. Briefly, the bacteria were grown in MRS broth for $24 \mathrm{~h}$. The L. paracasei culture was centrifuged at $12,000 \times g$ for $10 \mathrm{~min}$, neutralised to $\mathrm{pH} 7.0$ with $1 \mathrm{~N} \mathrm{NaOH}$ and concentrated tenfold by lyophilisation. The CFS was filter-sterilised using $0.22-\mu \mathrm{m}$ pore size filters (Minisart hydrophilic syringe filter; Sartorius Stedim Biotech GmbH, Goettingen, Germany) and stored at $-20^{\circ} \mathrm{C}$ until use. The supernatant was added at $7 \% \mathrm{v} / \mathrm{v}$.

\section{Preparation of epithelial cell monolayers}

Caco-2 cells were cultured in Dulbecco's modified Eagle's medium (DMEM) (Sigma-Aldrich) supplemented with $10 \%$ inactivated FBS, $1 \%$ glutamine, penicillin G $(0.1 \mathrm{U} / \mathrm{ml})$, and streptomycin $(0.1 \mathrm{mg} / \mathrm{ml})$. The cells were cultured at $37^{\circ} \mathrm{C}$ in an atmosphere of $5 \% \mathrm{CO}_{2}$ and 95\% air. Caco-2 cells were seeded in the upper chamber of a transwell filter $(3 \mu \mathrm{m}$ pore size, $6.5 \mathrm{~mm}$ diameter; Corning, NY) and incubated for 15-21 days. The cells were grown to confluence (until the trans-epithelial resistance (TER) reached $300 \Omega \mathrm{cm}^{2}$ ).

\section{Dendritic cells}

Human intestinal-like DCs generated from umbilical cord blood $\mathrm{CD}^{+} 4^{+}$progenitor cells (haematopoietic stem cells) were supplied by MatTek Corporation (Ashland, MA) and maintained according to the supplier's instructions [15].
Co-culture of Caco-2 cells and intestinal-like DCs and incubation with $L$. paracasei or its cell-free supernatant and S. typhi

Transwell inserts containing adherent monolayers of Caco-2 cells were positioned upside down in a 6-well plate, and $5 \times 10^{4}$ DCs were placed on the membrane. The DCs were allowed to adhere to the filters at $37^{\circ} \mathrm{C}$. After $4 \mathrm{~h}$, the transwell inserts were turned right side up and placed in 24-well plates containing $3 \times 10^{5} \mathrm{DCs} /$ well in the lower chamber. L. paracasei CNCM I-4034 $\left(10^{7} \mathrm{CFU} / \mathrm{ml}\right)$, its CFS or S. typhi CECT $725\left(10^{6} \mathrm{CFU} /\right.$ $\mathrm{ml}$ ) were added from the apical surface (top chamber), and the cells were incubated for $4 \mathrm{~h}$. Combinations of the probiotic or its CFS with $S$. typhi were prepared similarly. For the incubations, fresh cytokine and antibiotic-containing medium (DC-MM) was replaced with RPMI-1640 medium. After incubation, the bacteria or CFS were washed out of the wells, and the medium was replaced with DC-MM. After $20 \mathrm{~h}$, the culture supernatants were collected from the lower chamber for cytokine analysis, and the DCs in that compartment were collected for RNA extraction.

LPS at $20 \mathrm{ng} / \mathrm{ml}$ (Sigma-Aldrich) was used as a positive control. Negative control cultures contained unstimulated co-cultured DCs and Caco-2 cells.

\section{Cytokine and chemokine quantification in culture supernatants}

IL-1 $\beta$, IL-6, IL-8, IL-10, IL-12 (p40), IL-12 (p70), TNF- $\alpha$, IFN- $\gamma$, MCP-1/CCL2, MIP-1 $\alpha / C C L 3$, RANTES/CCL5, MDC/CCL22, IP-10/CXCL10 and TGF- $\beta$ were measured by immunoassay with a MILLIplex ${ }^{\text {тм }}$ kit (Linco Research Inc, Missouri, USA) using the Luminex 200 
system according to the manufacturer's instructions. Three independent experiments were performed.

\section{Extraction of RNA from DCs and evaluation of the expression of selected genes of the TLR signaling pathway}

Briefly, DCs collected from the lower chamber were lysed, and total RNA was extracted using the RNAqueous Kit (Ambion, Paisley, United Kingdom) and Turbo DNase treatment (Ambion) according to the manufacturers' recommendations. The quality of the RNA was verified on a Model 2100 Bioanalyzer (Agilent, Santa Clara, USA), and the RNA concentration was determined using a Rediplate 96 Ribogreen RNA Quantitation Kit (Gibco Invitrogen). Three samples were taken per treatment.

Real time RT-PCR analysis was carried out using a Human TLR Signaling Pathway PCR Array (SABiosciences Corporation, Frederick, Maryland, USA), which includes primer pairs specific for 20 selected genes related to TLR-mediated signaling pathways, as previously described by Bermudez-Brito et al. [14]. The expression level of each gene was analysed with $\mathrm{RT}_{2}$ Profiler PCR Array Data Analysis software (version 3.4; SABiosciences). The change in expression of each gene was reported as the fold change in expression (Fc). The results reflect logarithmic fold increase relative to the control samples.

Finally, IECs collected from the transwell membrane were lysed, and total RNA was extracted using the same method as described above. Real time RT-PCR analysis was carried out using a Human TLR Signaling Pathway PCR Array. The results are provided as [Additional files $1,2,3$ and 4].

\section{Statistical analysis}

All data are expressed as the mean \pm standard error of the mean (SEM) of three independent experiments. A two-sided Mann-Whitney $U$-test was used to determine changes in cytokine release and Fc gene differences between treatments. Statistical calculations were performed using NCSS 2007 software (Kaysville, UT). Differences between the treated cells and the controls were considered statistically significant when the $\mathrm{P}$ values were less than $0.05{ }^{*}$ ).

\section{Results}

Live L. paracasei CNCM I -4034 is a potent inducer of cytokine production

We first studied the effects of the probiotic strain and its CFS on cytokine and chemokine production in cocultures of human DCs and IECs. As shown in Figures 2, 3 and 4, we found that live L. paracasei CNCM I-4034 was a potent inducer of the pro-inflammatory cytokines
IL- $1 \beta$ and IL- 6 and the chemokine IL-8. In addition, we found that $L$. paracasei CNCM I-4034 increased the production of TGF- $\beta 2$, RANTES/CCL5 and IP-10/ CXCL10. In contrast, it decreased IL-12p40 (Figure 2), IL-10, TGF- $\beta 1$ (Figure 3) and MIP-1 $\alpha$ (Figure 4) levels but had no effect on IL-12p70 (Figure 2) levels or on the levels of any of the other chemokines tested (Figure 4).

As expected, LPS stimulation (the positive control) induced strong production of IL-6 and IL-8 (Figure 2). TNF- $\alpha$ was also induced; however, the difference never reached statistical significance (Figure 3). LPS did not induce chemokine release (Figure 4).

When we analysed the cytokines produced by cocultures exposed to $L$. paracasei CFS, we found that the cells were not very responsive to the CFS (Figures 2, 3 and 4). CFS-treated co-cultures exhibited a cytokine and chemokine profile similar to that of negative controls (non-exposed co-cultures) but showed less induction of TGF- $\beta 1$ and $\beta 2$ (Figure 3) and IP-10 (Figure 4).

\section{Live probiotic $L$. paracasei notably increases the release of cytokines and chemokines in the presence of Salmonella}

With respect to cytokine production, incubation of the co-culture with Salmonella alone did not increase the secretion of pro-inflammatory cytokines or chemokines (Figures 2, 3 and 4); only IL-6, IL-10, TGF- $\beta 1$ and MIP$1 \alpha$ levels were slightly modified.

As shown in Figures 2, 3 and 4, co-treatment with live L. paracasei CNCM I-4034 and Salmonella induced the release of a number of cytokines such as IL- 6 and TNF- $\alpha$ and of chemokines such as MIP- $1 \alpha /$ CCL3 and RANTES/CCL5; however, it induced less TGF- $\beta 2$. Furthermore, this treatment increased IL-10 production. No effects on MCP-1/CCL2, MDC/CCL22, TGF- $\beta 1$ or IL12p70 levels were observed.

In contrast, incubation with $L$. paracasei CFS and $S$. typhi resulted in increased release of the pro-inflammatory cytokines IL-1 $\beta$, IL-6 and IL-8 (Figure 2), TGF- $\beta 2$ (Figure 3), RANTES/CCL5 and IP-10/CXCL10 production (Figure 4 ) and decreased TGF- $\beta 1$ production (Figure 3).

\section{L. paracasei and its supernatant induce distinct strain-specific expression profiles of TLR signaling pathway molecules in human DCs}

Remarkably, most of the TLR signaling pathway genes tested in DCs were up-regulated upon incubation of the co-culture with $L$. paracasei CNCM I-4034 alone (Figures 5, 6, 7 and 8). The probiotic strain augmented TLR1, TLR4, TLR5 and TLR9 gene expression (Figures 5 and 6). In addition, the live probiotic induced upregulation of TOLLIP (Figure 6), CASP8 (Figure 7) and IL-10 (Figure 8). 


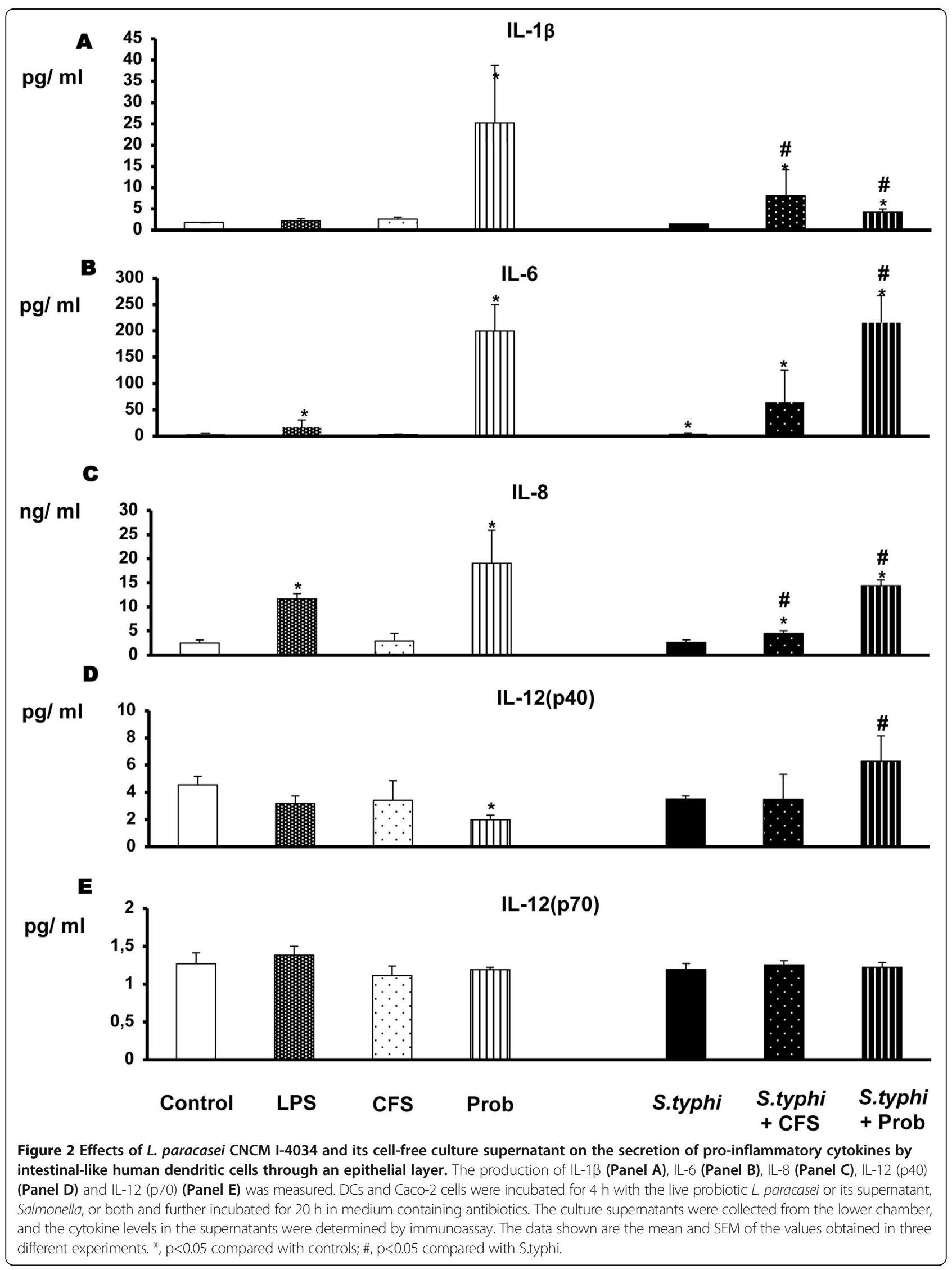


IL-10

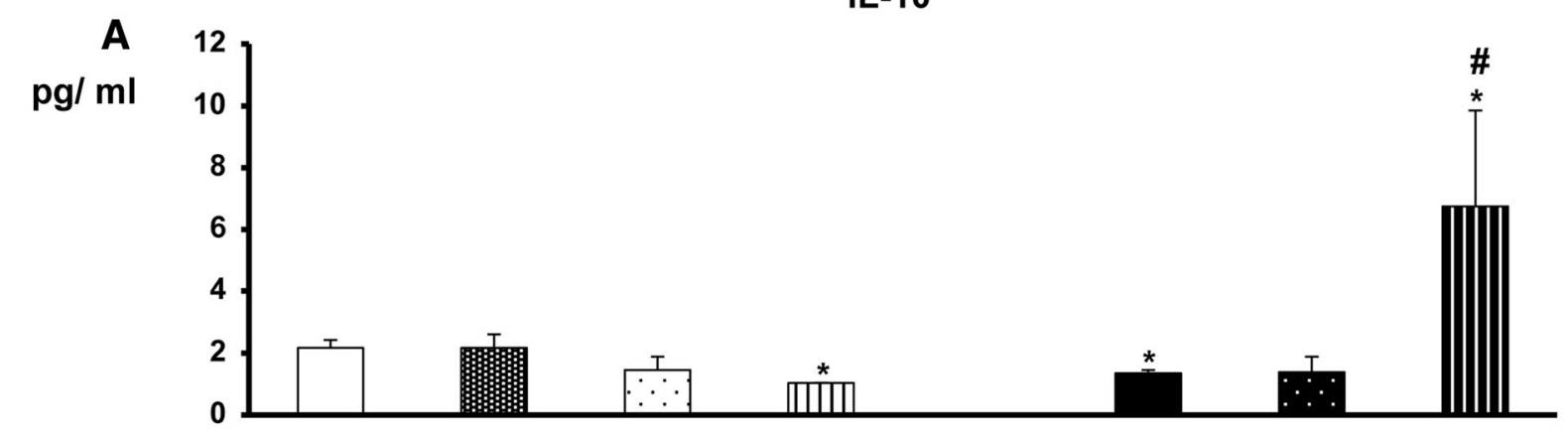

B

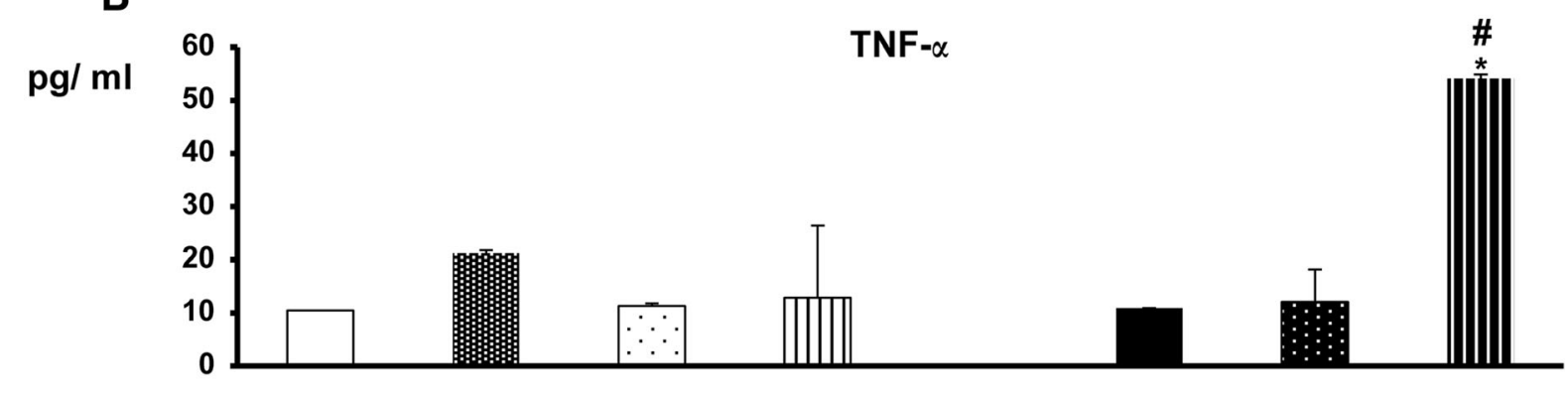

C

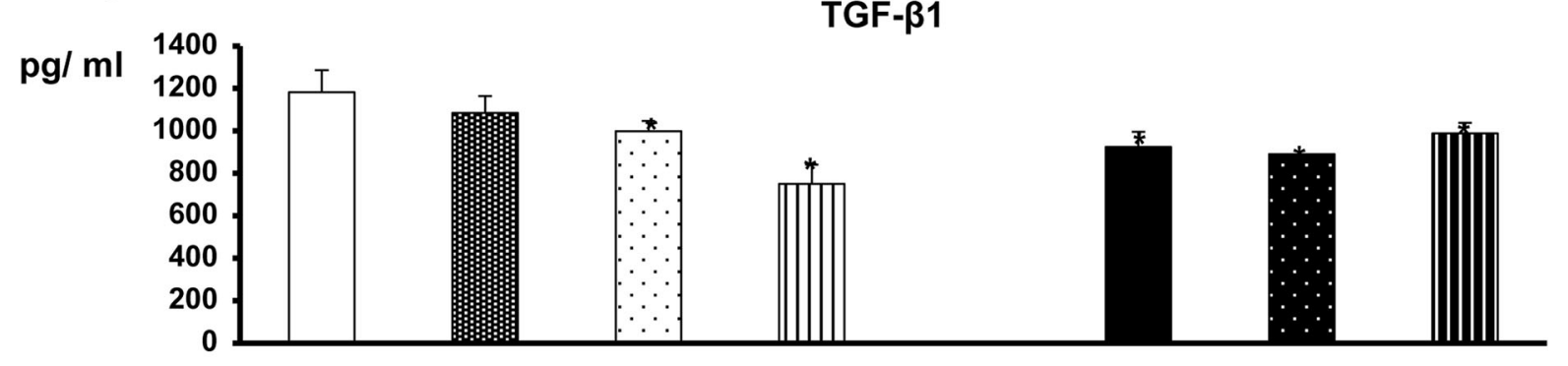

D

TGF- $\beta 2$

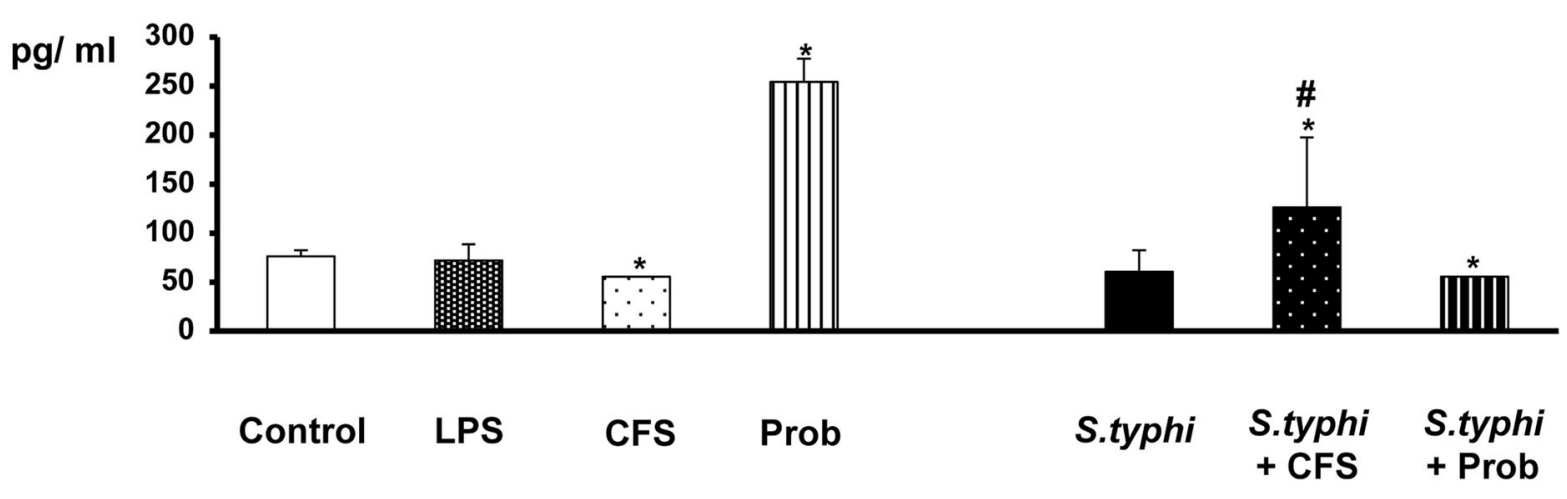

Figure 3 Measurement of anti-inflammatory cytokines and TNF- $\alpha$ in DCs through an epithelial layer after exposure to $L$. paracasei, Salmonella or a combination of the two. The production of IL-10 (Panel A), TNF-a (Panel B), TGF- $\beta 1$ (Panel C) and TGF- $\beta 2$ (Panel D) was measured. DCs and Caco-2 cells were incubated for $4 \mathrm{~h}$ with the live probiotic L. paracasei or its supernatant, Salmonella or both and further incubated for $20 \mathrm{~h}$ in medium containing antibiotics. The culture supernatants were collected from the lower chamber, and the cytokine levels were determined by immunoassay. The data shown are the mean and SEM of the values obtained in three different experiments. *, p<0.05 compared with controls; \#, p $<0.05$ compared with S.typhi; N.D. indicates not detected. 


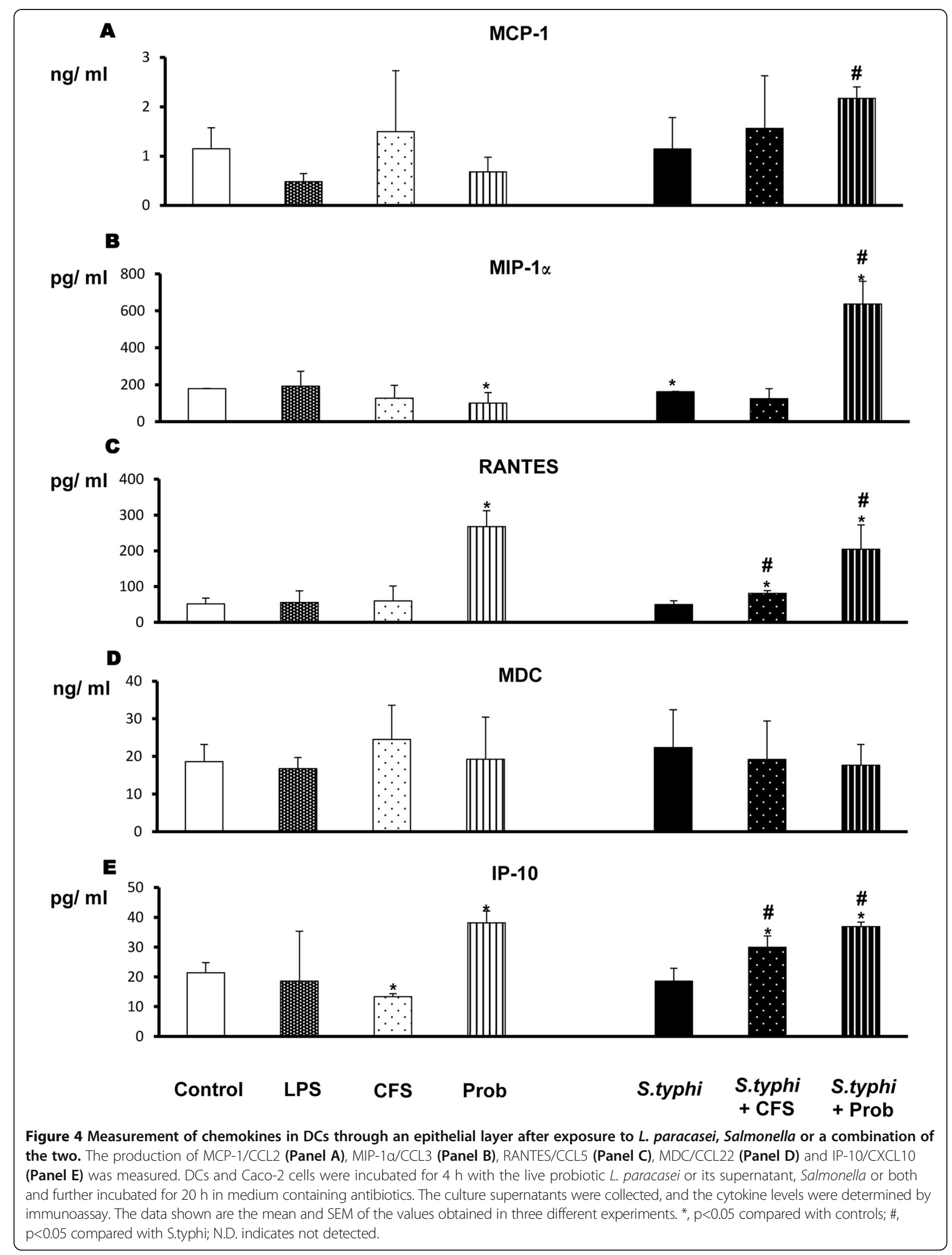



A
TLR1

Fc

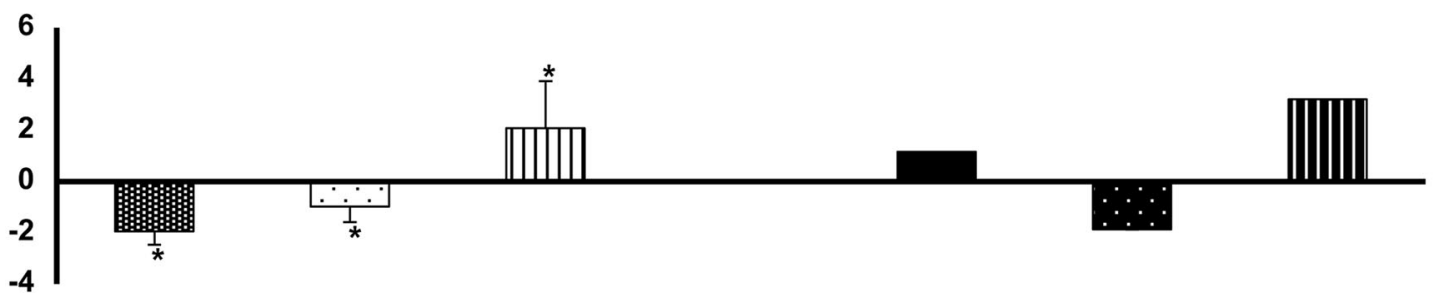

B

TLR2

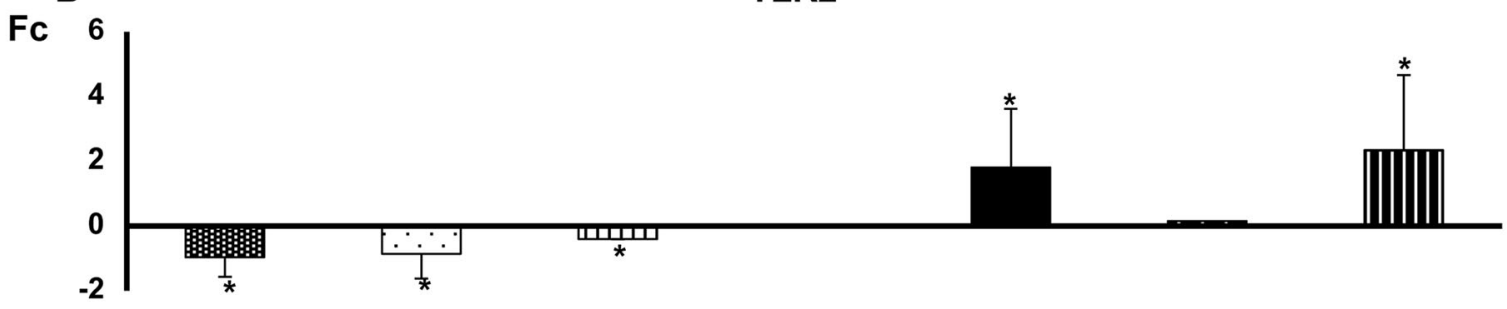

TLR3

C

N.D.

N.D.

Fc

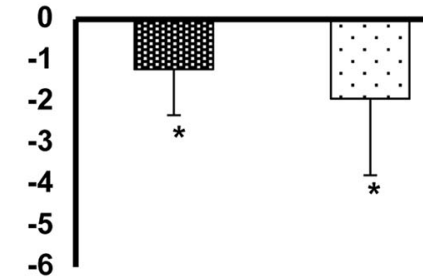

D

20

TLR4

Fc

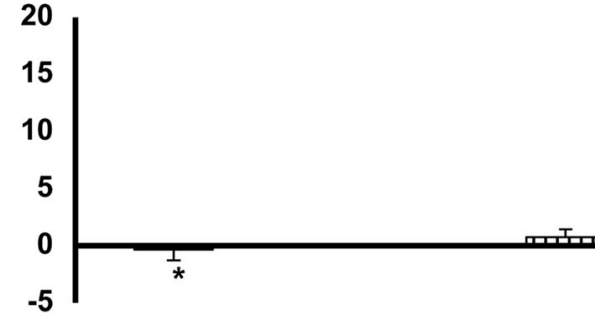

E

TLR5

FC

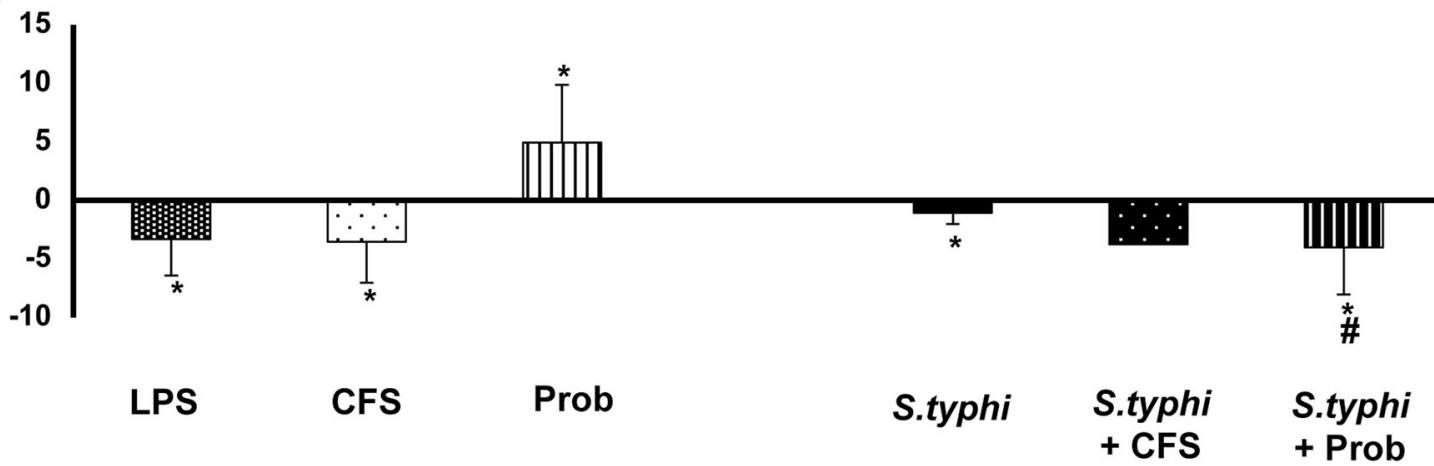

Figure 5 Expression of TLR genes in DCs in the presence of $L$. paracasei, Salmonella or a combination of the two. Comparison of the expression of TLR1 (Panel A), TLR2 (Panel B), TLR3 (Panel C), TLR4 (Panel D) and TLR5 (Panel E) in dendritic cells (DCs) taken from the lower chamber of a transwell co-culture model in the presence of the live probiotic L. paracasei or its supernatant, Salmonella or both. The fold change $(\mathrm{Fc})$ represents the ratio of the expression in the treated DCs to that of expression in the control cells. ${ }^{*}, \mathrm{p}<0.05$ compared with controls; \#, $p<0.05$ compared with S.typhi; N.D. indicates not detected. 
A

TLR9

Fc

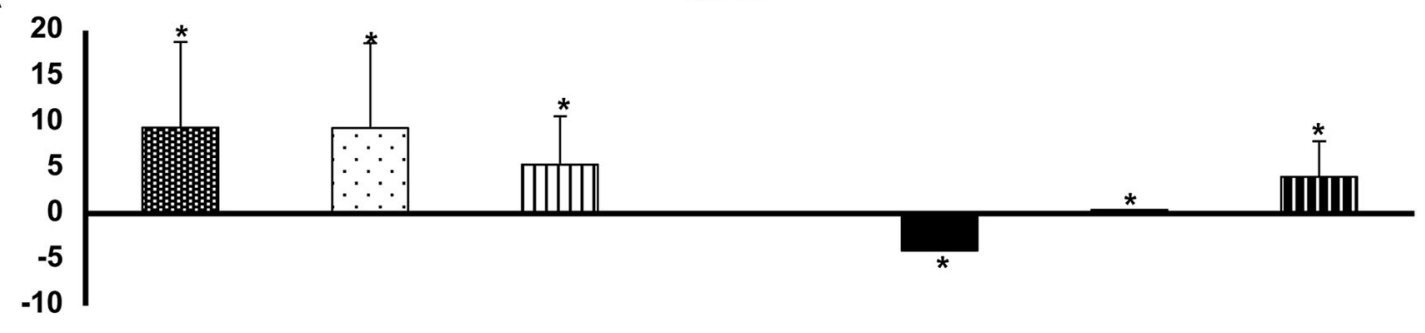

B

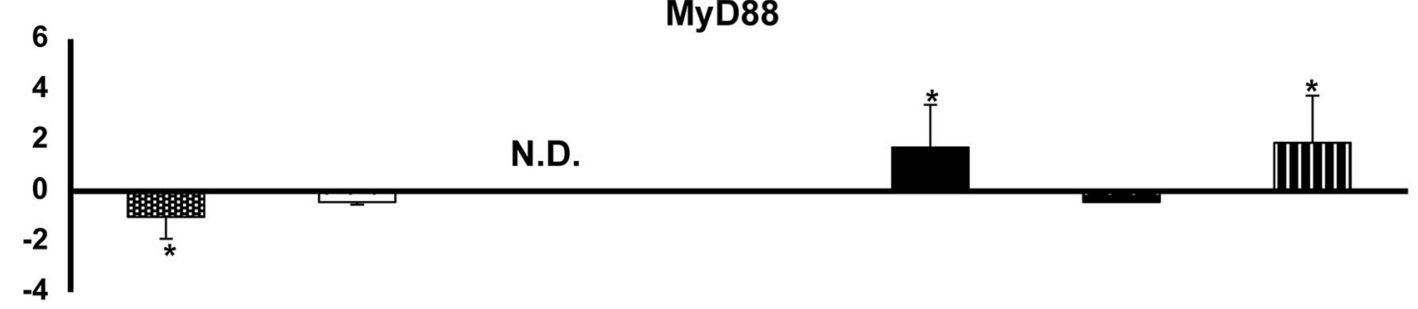

C

IRAK4

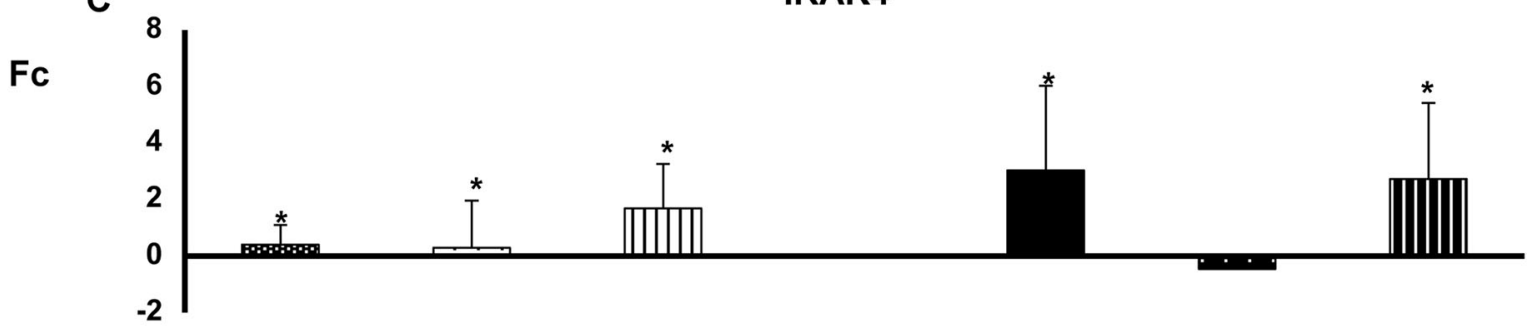

D

IRAK1

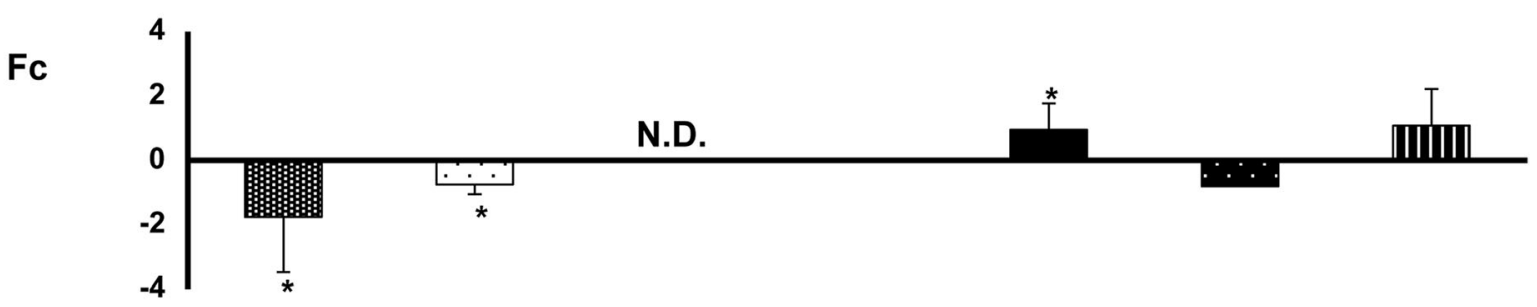

TOLLIP

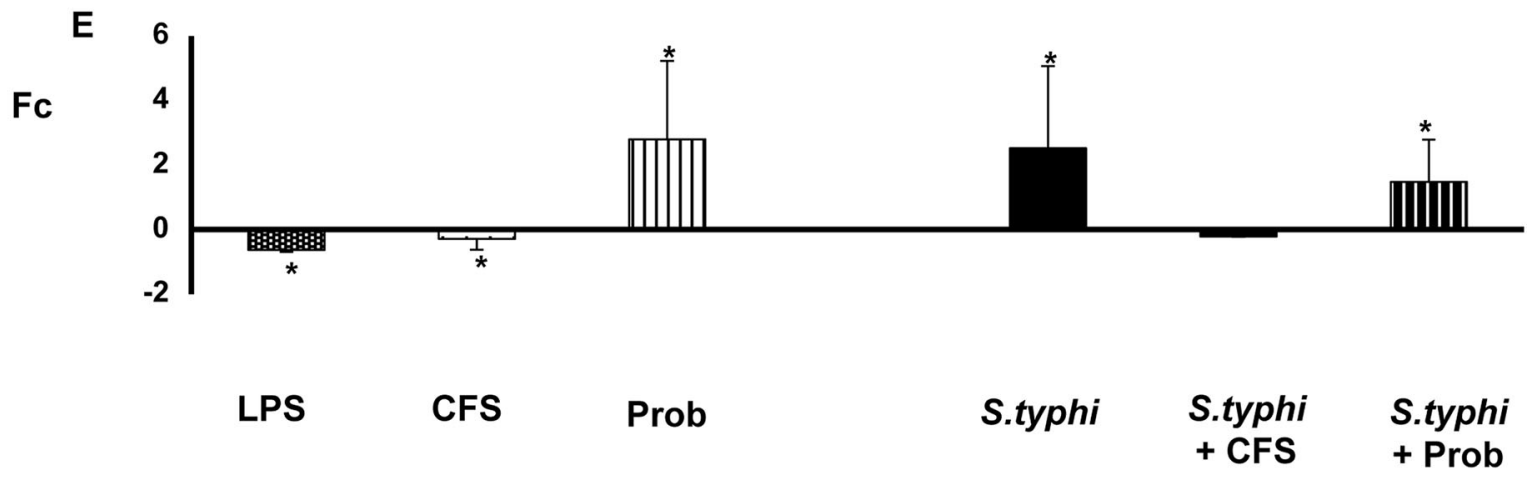

Figure 6 Expression of TLR signaling pathway components in DCs treated with L. paracasei, Salmonella or a combination of the two. Comparison of the expression of TLR9 (Panel A), MYD88 (Panel B), IRAK-1 (Panel C), IRAK-4 (Panel D) and TOLLIP (Panel E) in dendritic cells (DCs) taken from the lower chamber of a transwell co-culture model in the presence of the live probiotic L. paracasei or its supernatant, Salmonella or both. The fold change $(\mathrm{Fc})$ represents the ratio of the expression in the treated DCs to that of expression in the control cells. ${ }^{*}, p<0.05$ compared with controls; \#, p<0.05 compared with S.typhi; N.D. indicates not detected. 


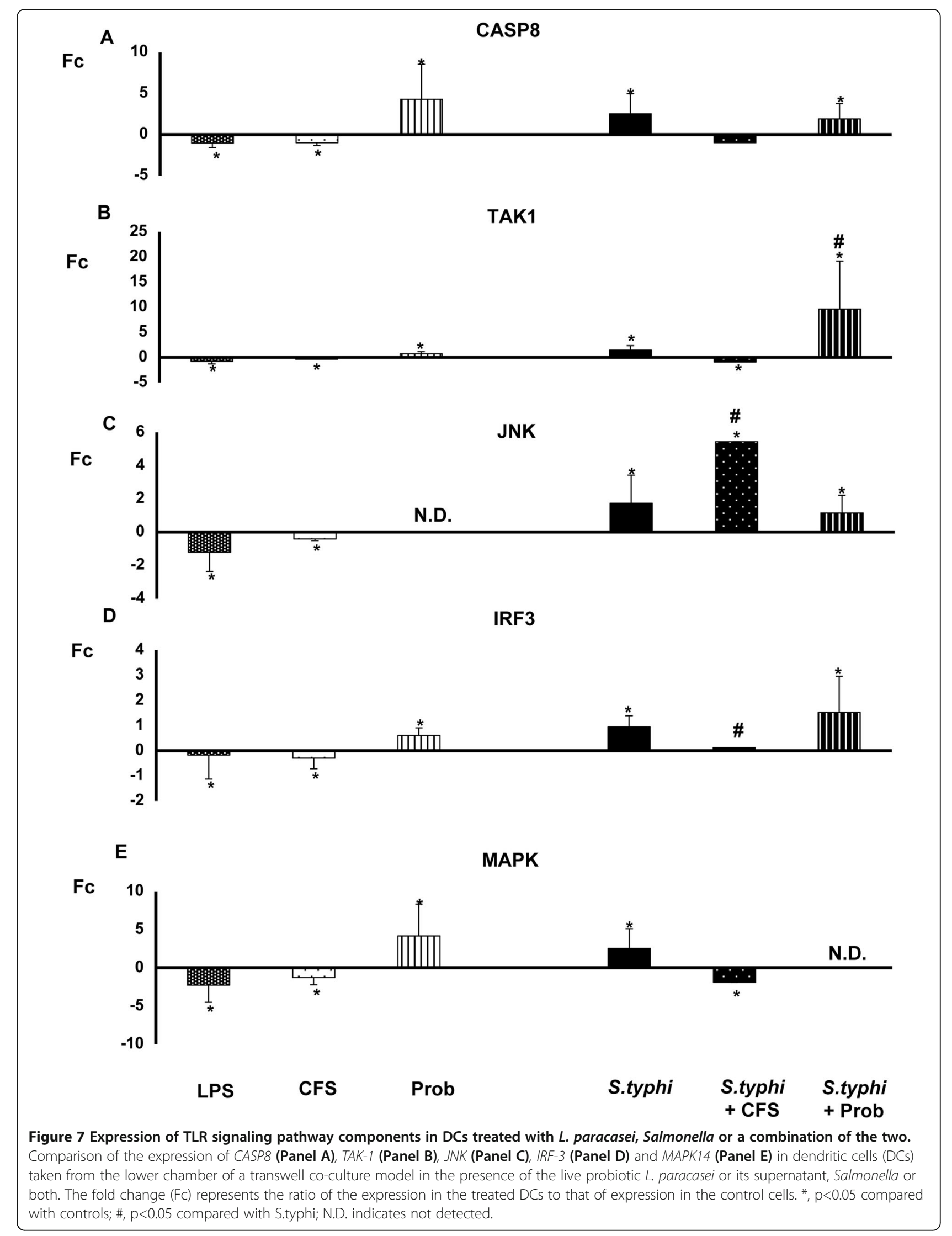




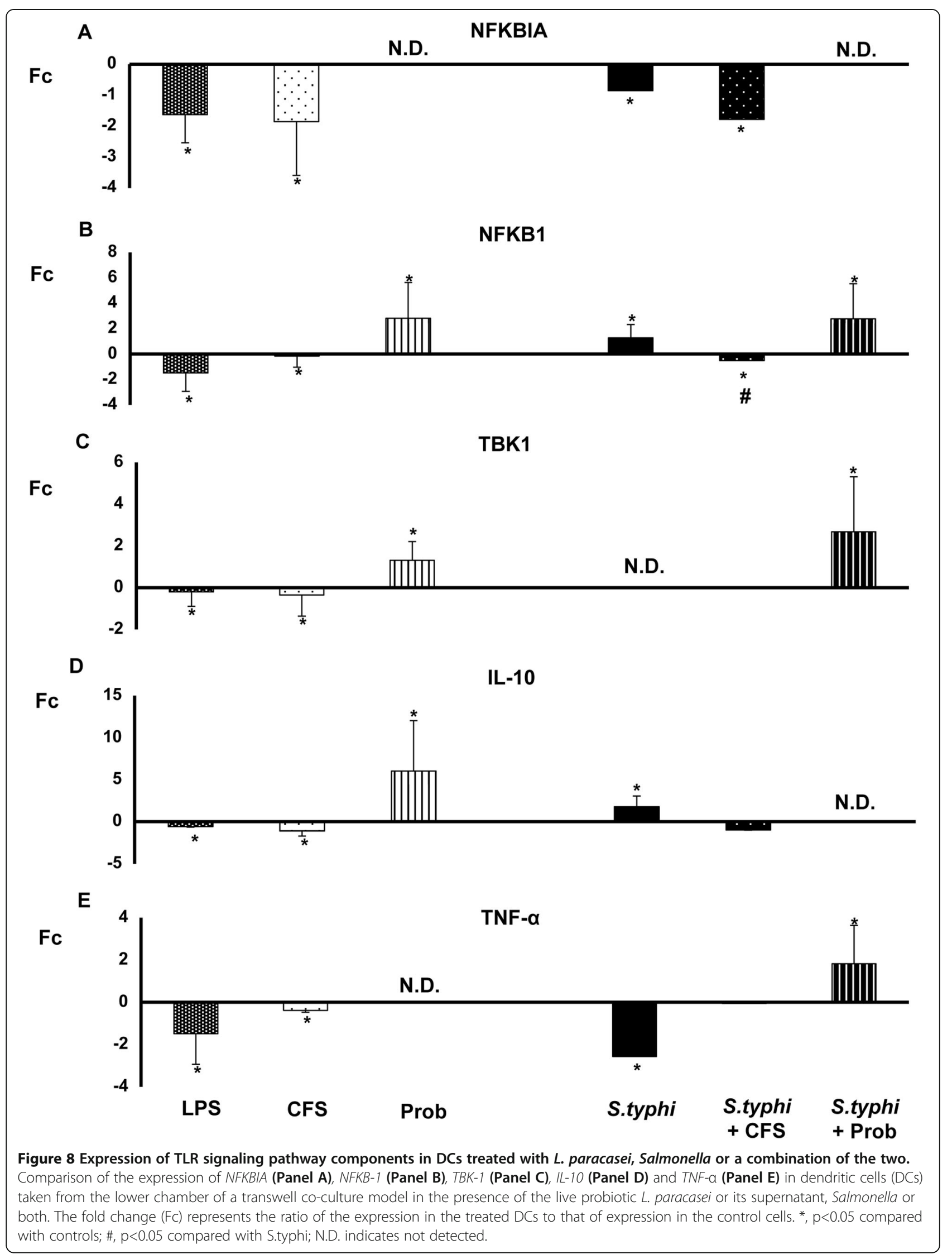


In contrast, CFS alone inhibited the expression of all DC TLR-selected genes except TLR9, which was upregulated, and $I R A K 4$, the expression of which was slightly increased (Figures 5 and 6).

When DCs and Caco-2 cells were co-cultured with live probiotic L. paracasei and Salmonella, strong and sustained transcription of most of the genes associated with TLR signaling pathways was observed in the human DCs (Figures 5, 6, 7 and 8). TLR1, TLR2, TLR4 and TLR9 gene expression was increased. Interestingly, in the presence of the enteropathogen, exposure to the live probiotic up-regulated $M Y D 88$ (Figure 6), CASP8 (Figure 7), NFKB1 and TBK1 (Figure 8) as well as other genes. Remarkably, the presence of the probiotic strain and Salmonella induced strong up-regulation of TAK1 (Figure 7).

Interestingly, simultaneous incubation of the coculture with L. paracasei CFS and Salmonella exerted a similar effect on TLR gene expression to that of CFS alone except for $J N K$ expression, which was strongly upregulated (Figure 7). Both CFS alone and CFS and Salmonella treatments decreased NFKBIA gene expression (Figure 8).

Analysis of TLR signaling pathway genes of Caco-2 cells collected from the transwell membrane is provided as Additional files 1, 2, 3 and 4 .

\section{Discussion}

To the best of our knowledge, this is the first study in which the immune response elicited by probiotics has been analysed using an in vitro bilayer system that includes human IECs growing on transwell membranes and human intestinal-like DCs developed from CD34+ progenitor cells isolated from umbilical cord blood. Moreover, in an attempt to mimic the in vivo conditions under which DCs are able to open tight junctions between adjacent epithelial cells and take up bacteria directly from the intestinal lumen [17], the transwell inserts were inverted and the human intestinal-like DCs were allowed to adhere to the IECs for $4 \mathrm{~h}[18,19]$. This culture system will facilitate functional studies, including studies of host-microbe interactions.

Live L. paracasei CNCM I-4034 alone was a potent inducer of IL-1 $\beta$, IL-6 and IL-8. Moreover, in the presence of Salmonella, L. paracasei induced the production of almost all the cytokines analysed including IL-6, IL-8 and TNF- $\alpha$, which are regulated by NF-kB [19], conversely to the results reported by our group in single cultures, either in Caco-2 cells [20] or in DCs [14], in which the probiotic L. paracasei down-regulated a broad array of proinflammatory cytokines in the presence of Salmonella. Altogether, the results suggest that $L$. paracasei enhances innate immunity via NF- $\mathrm{kB}$ activation rather than suppression. Furthermore, the stimulation of other innate immunity pathways, i.e., ERK mediated IL-10 production and TGF- $\beta$ production mediated by P38 MAPK, ERK, and JNK, could also be involved [21,22]. This activation of innate immunity may help strengthen intestinal defence and epithelial barrier function.

TGF- $\beta$ is considered a key immunoregulatory factor in promoting immunoglobulin A (IgA) production [23] and maintaining immune tolerance [24,25]. In this context, live $L$. paracasei stimulated TGF- $\beta 2$ release. Furthermore, CFS is a potent inducer of TGF- $\beta 2$ in response to $S$. typhi. Immunomodulatory effects of TGF- $\beta 2$ have been reported in conditions such as atopy and systemic inflammatory response syndrome [26,27]. In addition, IL-6 was notably secreted upon stimulation of cells with live $L$. paracasei alone or with $L$. paracasei and its CFS in the presence of Salmonella. Probiotic strains have been described as potent inducers of IL-6 [28] and enhancers of IgA responses [29]. Uematsu et al. [30] proposed that commensal bacteria induce IgA production through a mechanism mediated by TLR5, an interpretation that is consistent with our results. Altogether, these results suggest that L.paracasei could lead to the generation of $\operatorname{IgA}$ and reinforce epithelial barrier function because secreted IgA limits bacterial association with the intestinal epithelial surface and restricts the penetration of pathogens across the gut epithelium [31], precluding a systemic immune response [32]. Interestingly, this argument is strengthened by our recent observation that Bifidobacterium breve CNCM I-4035 administration increases secretory IgA content after 30-day intervention in healthy volunteers [33].

In contrast, IECs and DCs were poorly responsive to $S$. typhi, contrary to the results reported by Zoumpopoulou et al. [1] and Vossenkämper et al. [19]. In addition, S. typhi was unable to induce the release of chemokines such as RANTES and induced low levels of MIP-1 $\alpha$. However, it is important to note that Salmonella did induce changes in the expression of TLR signaling pathway genes in human DCs. These changes in gene expression may be due to crossing of the monolayer by bacterial compounds or to direct contact between the pathogen and DCs that crept between the epithelial cells and contacted the pathogen directly from the luminal side. Taken together, our results suggest that Salmonella cells were not able to penetrate the epithelial layer and interact directly with the DCs. In addition, TLR genes expression in IECs was decreased in response of Salmonella stimuli [see Additional file 1]. Furthermore, Salmonella just induced the expression of $M y D 88, \operatorname{Casp} 8, T A K 1$ and NFKBIA in IECs [see Additional files 1, 2, 3 and 4]. Thus, the low cytokine profile observed in the co-cultured DCs and IECs could be due to the action of IECs and bacterial compounds that crossed the monolayer. Consequently, IECs not only form a physical barrier to 
bacteria but also regulate DC cytokine production, possibly through epithelial cell-derived factors [34,35], and could contribute to prevention of an exaggerated inflammation and thereby to the maintenance of immune homeostasis in the gut [36].

Interestingly, the culture supernatant exhibited activity when Salmonella was present, in agreement with our previous findings [14]. These data indicate that soluble bacterial product(s) released by $L$. paracasei $\mathrm{CNCM}$ I-4034 were able to cross the epithelial monolayer and activate the DCs in the lower chamber. Moreover, IECs and DCs are able to recognise probiotic secreted factor(s) without inducing inflammatory reactions [35]. This culture supernatant modulated the ability of the DCs to produce inflammatory cytokines in response to pathogens.

Previous results obtained by our group suggest that probiotics modulate the cytokine profile in immune cells through TLRs stimulation [14]. We therefore analysed the expression of TLRs in DCs taken from the lower chamber of our co-culture system. The first of these experiments showed that few genes were up-regulated by the supernatant in DCs. Exposure to CFS led to an overall decrease in expression of all the TLR genes except TLR9, which was strongly up-regulated. This upregulation was also found in our previous study in immune cells. Conversely, the expression of this TLR was decreased in IECs. Moreover, the expression of TLR1, TLR3 and TLR4 was up-regulated upon CFS stimulation [see Additional files 1 and 2]. Because CFS by itself did not induce cytokine production, TLR9 may have a key role in the context of probiotic-host interactions, and the results suggest that the immunomodulatory effects of $L$. paracasei product(s) may involve mechanisms that overlap with those associated with CpG [14,2]. Moreover, down-regulation of TLRs genes may block the production of pro-inflammatory cytokines. Interestingly, JNK gene expression in DCs was notably increased with this treatment, whereas in IECs was down-regulated [see Additional file 3]. JNK phosphorylation leads to the activation of transcription factors such as NF- $\mathrm{kB}$, which directs the production of pro-inflammatory cytokines [37], consistent with our results. Overall, it appears that in the presence of infectious agents, the supernatant of L. paracasei promotes cytokine production and thereby enables the cells to combat pathogens.

In the presence of Salmonella and the live probiotic, TLR2 and TLR9 were slightly up-regulated in DCs, whereas TLR1, TLR2, TLR3 and TLR4 were increased in IECs. Like the results of several previous studies, our results suggest that exposure to live $L$. paracasei increases the expression of TLR2 and TLR9, activating innate immunity in immune cells [38-40]. Furthermore,
TLR2 stimulation appears to enhance the epithelial barrier, and it has been shown that activation of this TLR plays an essential role in resistance to bacterial invasion. Several studies have described increased expression of TLR9 after the administration of probiotics such as $L$. johnsonii [41]. In this line, and according with our results, it has been shown a protective role for TLR1 during bacterial infection via its signaling in the intestinal epithelium [42]. TLR4 was also up-regulated in our experiments in DCs and IECs. TLR4 can recognise LPS present in the walls of Gram-negative bacteria [43] and plays a key role in defence against Salmonella [30]. In the presence of Salmonella, TNF- $\alpha$ gene expression is up-regulated, a finding that correlates with the observed cytokine profile. Altogether, these results suggest that when pathogens are present, $L$. paracasei promotes the local stimulation of innate immune responses in the gut through the activation of NF- $\mathrm{BB}$, thus preventing systemic inflammation. Follow-up studies are ongoing to evaluate whether this probiotic strain can exert a protective effect in vivo in a murine model.

\section{Conclusions}

This study demonstrates that the effects of probiotics should be studied on complex of cells because cultures of single cell types do not exhibit the effects of the exposure to a probiotic strain. DCs react differently to probiotics and pathogens in the presence and absence of IECs. Co-culture models such as the one presented here will facilitate the study of host-microbe interactions. Moreover, this system also provides a feasible platform for the development of novel probiotics or other agents for further animal studies and clinical trials.

\section{Additional files}

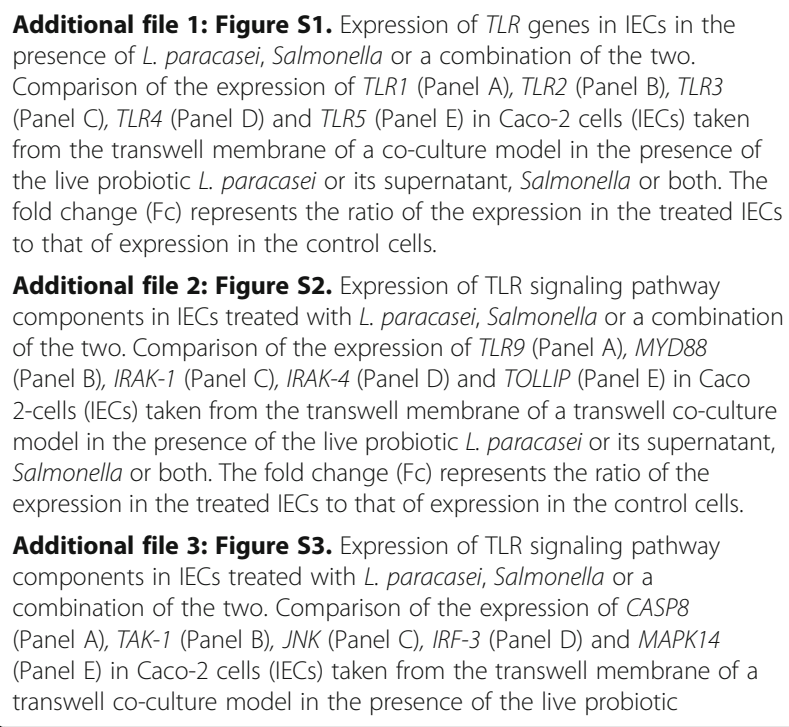

Additional file 1: Figure S1. Expression of TLR genes in IECS in the presence of $L$. paracasei, Salmonella or a combination of the two. Comparison of the expression of TLR1 (Panel A), TLR2 (Panel B), TLR3 (Panel C), TLR4 (Panel D) and TLR5 (Panel E) in Caco-2 cells (IECS) taken from the transwell membrane of a co-culture model in the presence of the live probiotic L. paracasei or its supernatant, Salmonella or both. The fold change (FC) represents the ratio of the expression in the treated IECS to that of expression in the control cells.

Additional file 2: Figure S2. Expression of TLR signaling pathway components in IECs treated with L. paracasei, Salmonella or a combination of the two. Comparison of the expression of TLR9 (Panel A), MYD88 (Panel B), IRAK-1 (Panel C), IRAK-4 (Panel D) and TOLLIP (Panel E) in Caco 2-cells (IECS) taken from the transwell membrane of a transwell co-culture model in the presence of the live probiotic L. paracasei or its supernatant, Salmonella or both. The fold change (Fc) represents the ratio of the expression in the treated IECS to that of expression in the control cells.

Additional file 3: Figure S3. Expression of TLR signaling pathway components in IECs treated with L. paracasei, Salmonella or a combination of the two. Comparison of the expression of CASP8 (Panel A), TAK-1 (Panel B), JNK (Panel C), IRF-3 (Panel D) and MAPK14 (Panel E) in Caco-2 cells (IECS) taken from the transwell membrane of a transwell co-culture model in the presence of the live probiotic 
L. paracasei or its supernatant, Salmonella or both. The fold change (FC) represents the ratio of the expression in the treated IECS to that of expression in the control cells.

Additional file 4: Figure S4. Expression of TLR signaling pathway components in IECs treated with L. paracasei, Salmonella or a combination of the two. Comparison of the expression of NFKBIA (Panel A), NFKB-1 (Panel B), TBK-1 (Panel C), LL-10 (Panel D) and TNF-a (Panel E) in Caco-2 cells (IECS) taken from the transwell membrane of a transwell co-culture model in the presence of the live probiotic $L$. paracasei or its supernatant, Salmonella or both. The fold change (Fc) represents the ratio of the expression in the treated IECS to that of expression in the control cells.

\section{Competing interests}

EM and FR are members of the Hero Global Technology Center, Hero Spain, S.A. The sponsor had no role in the biological sample analysis, statistical analysis or data interpretation. This does not alter our adherence to all the BMC Microbiology policies on sharing data and materials.

\section{Authors' contributions}

Conceived and designed the experiments: AG CGLL. Performed the experiments: MBB SMQ. Analyzed the data: MBB. Contributed reagents/ materials/analysis tools: EM FR. Wrote the paper: MBB AG. All authors read and approved the final manuscript.

\section{Acknowledgements}

CGLL is the recipient of a postdoctoral fellowship from Plan Propio at the University of Granada.

\section{Author details}

${ }^{1}$ Institute of Nutrition and Food Technology "José Mataix", Department of Biochemistry and Molecular Biology II, University of Granada, Biomedical Research Center, Avenida del Conocimiento s/n, 18100 Armilla, Granada, Spain. ${ }^{2}$ Hero Global Technology Center, Hero Spain, S.A., 30820 Alcantarilla, Murcia, Spain.

\section{Received: 27 January 2015 Accepted: 11 March 2015}

Published online: 01 April 2015

\section{References}

1. Zoumpopoulou G, Tsakalidou E, Dewulf J, Pot B, Grangette C. Differential crosstalk between epithelial cells, dendritic cells and bacteria in a co-culture model. Int J Food Microbiol. 2009;131:40-51.

2. Bermudez-Brito M, Plaza-Díaz J, Muñoz-Quezada S, Gomez-Llorente C, Gil A. Mechanisms of action of probiotics. Ann Nutr Metab. 2012;61:160-74.

3. Meijerink M, Wells JM. Probiotic modulation of dendritic cells and T cell responses in the intestine. Benef Microbes. 2010;1:317-26.

4. Hörmannsperger $G$, Haller D. Molecular crosstalk of probiotic bacteria with the intestinal immune system: clinical relevance in the context of inflammatory bowel disease. Int J Med Microbiol. 2010;300:63-73.

5. Butler M, Ng CY, Van Heel DA, Lombardi G, Lechler R, Playford RJ, et al. Modulation of dendritic cell phenotype and function in an in vitro model of the intestinal epithelium. Eur J Immunol. 2006;36:864-74.

6. Rimoldi M, Chieppa M, Salucci V, Avogadri F, Sonzogni A, Sampietro GM, et al. Intestinal immune homeostasis is regulated by the crosstalk between epithelial cells and dendritic cells. Nat Immunol. 2005;6:507-14.

7. Badia R, Brufau MT, Guerrero-Zamora AM, Lizardo R, Dobrescu I, MartinVenegas $R$, et al. $\beta$-Galactomannan and Saccharomyces cerevisiae var. boulardii modulate the immune response against Salmonella enterica serovar Typhimurium in porcine intestinal epithelial and dendritic cells. Clin Vaccine Immunol. 2012;19:368-76.

8. Swart AL, Hensel M. Interactions of Salmonella enterica with dendritic cells. Virulence. 2012;3:660-7.

9. Muñoz-Quezada S, Chenoll E, Vieites Fernández JM, Genovés S, Maldonado J, Bermúdez-Brito M, et al. Isolation, identification and characterization of three novel probiotic strains (Lactobacillus paracasei CNCM 1-4034, Bifidobacterium breve CNCM I-4035 and Lactobacillus rhamnosus CNCM I-4036) from faeces of exclusively breast milk fed infants. Br J Nutr. 2013;109:S51-62.

10. Muñoz-Quezada S, Bermudez-Brito M, Chenoll E, Genovés S, Gomez-Llorente C, Plaza-Diaz J, et al. Competitive inhibition of three novel bacteria isolated from faeces of breast milk-fed infants against selected enteropathogens. Br J Nutr. 2013;109 Suppl 2:S63-9.

11. Luongo D, Miyamoto J, Bergamo P, Nazzaro F, Baruzzi F, Sashihara T, et al. Differential modulation of innate immunity in vitro by probiotic strains of Lactobacillus gasseri. BMC Microbiol. 2013;13:298.

12. Haller D, Serrant P, Peruisseau G, Bode C, Hammes WP, Schiffrin E, et al. IL-10 producing CD14low monocytes inhibit lymphocyte-dependent activation of intestinal epithelial cells by commensal bacteria. Microbiol Immunol. 2002;46:195-205.

13. Tsilingiri K, Barbosa T, Penna G, Caprioli F, Sonzogni A, Viale G, et al. Probiotic and postbiotic activity in health and disease: comparison on a novel polarised ex-vivo organ culture model. Gut. 2012;61:1007-15.

14. Bermudez-Brito M, Muñoz-Quezada S, Gomez-Llorente C, Matencio E, Bernal MJ, Romero F, et al. Human intestinal dendritic cells decrease cytokine release against Salmonella infection in the presence of Lactobacillus paracasei upon TLR activation. PLoS One. 2012;7:e43197.

15. Ayehunie S, Snell M, Child M, Klausner M. A plasmacytoid dendritic cell (CD123+/CD11C-) based assay system to predict contact allergenicity of chemicals. Toxicology. 2009;264:1-9.

16. Adams CA. The probiotic paradox: live and dead cells are biological response modifiers. Nutr Res Rev. 2010;23:37-46.

17. Rescigno M, Urbano M, Valzasina B, Francolini M, Rotta G, Bonasio R, et al. Dendritic cells express tight junction proteins and penetrate gut epithelial monolayers to sample bacteria. Nat Immunol. 2001;2:361-7.

18. Rimoldi M, Chieppa M, Vulcano M, Allavena P, Rescigno M. Intestinal epithelial cells control dendritic cell function. Ann N Y Acad Sci. 2004;1029:66-74.

19. Vossenkämper A, Marchès O, Fairclough PD, Warnes G, Stagg AJ, Lindsay $J \mathrm{O}$, et al. Inhibition of NF-KB signaling in human dendritic cells by the enteropathogenic Escherichia coli effector protein NleE. J Immunol. 2010;185:4118-27.

20. Muñoz SI, Bermudez-Brito M, Gómez-Llorente C, Bernal MJ, Ortuño-Díaz I, Gil A. Lactobacillus paracasei CNCM I-4034 disminuye la respuesta inflamatoria inducida por Salmonella typhi en células caco-2. Nutr Hosp. 2012;27(Supl):66.

21. Pagnini C, Saeed R, Bamias G, Arseneau KO, Pizarro TT, Cominelli F. Probiotics promote gut health through stimulation of epithelial innate immunity. Proc Natl Acad Sci U S A. 2010;107:454-9.

22. Saraiva M, O'Garra A. The regulation of IL-10 production by immune cells. Nat Rev Immunol. 2010;10:170-81.

23. Nikniaz L, Ostadrahimi A, Mahdavi R, Hejazi MA, Hosseini Salekdeh G. Effects of synbiotic supplementation on breast milk levels of IgA, TGF- $\beta 1$, and TGF-ß2. J Hum Lact. 2013;29:591-6.

24. Kaplan DH, Li MO, Jenison MC, Shlomchik WD, Flavell RA, Shlomchik MJ. Autocrine/paracrine TGFbeta1 is required for the development of epidermal Langerhans cells. J Exp Med. 2007;204:2545-52.

25. Kushwah $\mathrm{R}, \mathrm{Hu} \mathrm{J}$. Role of dendritic cells in the induction of regulatory $T$ cells. Cell Biosci. 2011;1:20.

26. Hatsushika K, Hirota T, Harada M, Sakashita M, Kanzaki M, Takano S, et al. Transforming growth factor-beta (2) polymorphisms are associated with childhood atopic asthma. Clin Exp Allergy. 2007:37:1165-74.

27. Stoiser B, Knapp S, Thalhammer F, Locker GJ, Kofler J, Hollenstein U, et al. Time course of immunological markers in patients with the systemic inflammatory response syndrome: evaluation of sCD14, sVCAM-1, sELAM-1, MIP-1 alpha and TGF-beta 2. Eur J Clin Invest. 1998;28:672-8.

28. Weiss G, Christensen HR, Zeuthen LH, Vogensen FK, Jakobsen M, Frøkiær $H$ Lactobacilli and bifidobacteria induce differential interferon- $\beta$ profiles in dendritic cells. Cytokine. 2011;56:520-30.

29. Christensen HR, Frøkiaer H, Pestka JJ. Lactobacilli differentially modulate expression of cytokines and maturation surface markers in murine dendritic cells. J Immunol. 2002;168:171-8.

30. Uematsu S, Fujimoto $K$, Jang MH, Yang BG, Jung YJ, Nishiyama $M$, et al. Regulation of humoral and cellular gut immunity by lamina propria dendritic cells expressing Toll-like receptor 5. Nat Immunol. 2008;9:769-76.

31. Hooper LV, Macpherson AJ. Immune adaptations that maintain homeostasis with the intestinal microbiota. Nat Rev Immunol. 2010;10:159-69.

32. Feng $\mathrm{T}$, Elson CO, Cong Y. Treg cell-Ig A axis in maintenance of host immune homeostasis with microbiota. Int Immunopharmacol. 2011;11:589-92.

33. Plaza-Diaz J, Gomez-Llorente C, Campaña-Martin L, Matencio E, Ortuño I, Martínez-Silla R, et al. Safety and immunomodulatory effects of three probiotic strains isolated from the feces of breast-fed infants in healthy 
adults: SETOPROB study. PLoS One. 2013;8:e78111. doi:10.1371/ journal.pone.0078111.

34. Rimoldi M, Chieppa M, Larghi P, Vulcano M, Allavena P, Rescigno M. Monocyte-derived dendritic cells activated by bacteria or by bacteriastimulated epithelial cells are functionally different. Blood. 2005;106:2818-26.

35. Putaala H, Barrangou R, Leyer GJ, Ouwehand AC, Hansen EB, Romero DA et al. Analysis of the human intestinal epithelial cell transcriptional response to Lactobacillus acidophilus, Lactobacillus salivarius, Bifidobacterium lactis and Escherichia coli. Benef Microbes. 2010;1:283-95.

36. Westendorf AM, Fleissner D, Hansen W, Buer J. T cells, dendritic cells and epithelial cells in intestinal homeostasis. Int J Med Microbiol. 2010;300:11-8.

37. Lavelle EC, Murphy C, O'Neill LA, Creagh EM. The role of TLRs, NLRs, and RLRs in mucosal innate immunity and homeostasis. Mucosal Immunol. 2010;3:17-28.

38. Vizoso Pinto MG, Rodriguez Gomez M, Seifer S, Watzl B, Holzapfel WH, Franz CM. Lactobacilli stimulate the innate immune response and modulate the TLR expression of HT29 intestinal epithelial cells in vitro. Int J Food Microbiol. 2009;33:86-93.

39. Tao Y, Drabik KA, Waypa TS, Musch MW, Alverdy JC, Schneewind O, et al Soluble factors from Lactobacillus GG activate MAPKs and induce cytoprotective heat shock proteins in intestinal epithelial cells. Am J Physiol. 2006:290:1018-30.

40. Abreu MT, Fukata M, Arditi M. TLR signaling in the gut in health and disease. J Immunol. 2005;174:4453-60.

41. Sugiura Y, Kamdar K, Khakpour S, Young G, Karpus WJ, DePaolo RW. TLR1induced chemokine production is critical for mucosal immunity against Yersinia enterocolitica. Mucosal Immunol. 2013;6:1101-9.

42. Lin YP, Thibodeaux CH, Peña JA, Ferry GD, Versalovic J. Probiotic Lactobacillus reuteri suppress proinflammatory cytokines via c-Jun. Inflamm Bowel Dis. 2008;14:1068-83.

43. Castillo NA, Perdigón G, De Moreno De Leblanc A. Oral administration of a probiotic Lactobacillus modulates cytokine production and TLR expression improving the immune response against Salmonella enterica serovar typhimurium infection in mice. BMC Microbiol. 2011;11:177.

\section{Submit your next manuscript to BioMed Central and take full advantage of:}

- Convenient online submission

- Thorough peer review

- No space constraints or color figure charges

- Immediate publication on acceptance

- Inclusion in PubMed, CAS, Scopus and Google Scholar

- Research which is freely available for redistribution 